\title{
Intrauterine death following intraamniotic triiodothyronine and thyroxine therapy for fetal goitrous hypothyroidism associated with polyhydramnios and caused by a thyroglobulin mutation
}

\author{
Pradeep Vasudevan', Corrina Powell', Adeline K Nicholas², Ian Scudamore ${ }^{3}$, \\ James Greening ${ }^{4}$, Soo-Mi Park 5 and Nadia Schoenmakers ${ }^{2}$ \\ 'Leicester Clinical Genetics, Women's and Children's Services, Leicester Royal Infirmary, Leicester, UK, \\ 2University of Cambridge Metabolic Research Laboratories, Wellcome Trust-Medical Research Council \\ Institute of Metabolic Science, Addenbrooke's Hospital, Cambridge, UK, 3Department of Obstetrics and \\ Gynaecology, Women's and Prenatal Services, Leicester General Hospital, Leicester, UK, 'Department of \\ Paediatric Endocrinology, Leicester Royal Infirmary, Leicester, UK, and ${ }^{5}$ Department of Clinical Genetics, \\ Cambridge University Hospitals NHS Foundation Trust, Cambridge, UK
}

\author{
Correspondence \\ should be addressed \\ to $\mathrm{N}$ Schoenmakers \\ Email \\ naaa2@cam.ac.uk
}

\section{Summary}

In the absence of maternal thyroid disease or iodine deficiency, fetal goitre is rare and usually attributable to dyshormonogenesis, for which genetic ascertainment is not always undertaken in the UK. Mechanical complications include tracheal and oesophageal compression with resultant polyhydramnios, malpresentation at delivery and neonatal respiratory distress. We report an Indian kindred in which the proband (first-born son) had congenital hypothyroidism $(\mathrm{CH})$ without obvious neonatal goitre. His mother's second pregnancy was complicated by fetal hypothyroid goitre and polyhydramnios, prompting amniotic fluid drainage and intraamniotic therapy (with liothyronine, T3 and levothyroxine, T4). Sadly, intrauterine death occurred at 31 weeks. Genetic studies in the proband demonstrated compound heterozygous novel (c.5178delT, p.A1727Hfs*26) and previously described (c.7123G > A, p.G2375R) thyroglobulin (TG) mutations which are the likely cause of fetal goitre in the deceased sibling. TG mutations rarely cause fetal goitre, and management remains controversial due to the potential complications of intrauterine therapy however an amelioration in goitre size may be achieved with intraamniotic T4, and intraamniotic T3/T4 combination has achieved a favourable outcome in one case. A conservative approach, with surveillance, elective delivery and commencement of levothyroxine neonatally may also be justified, although intubation may be required post delivery for respiratory obstruction. Our observations highlight the lethality which may be associated with fetal goitre. Additionally, although this complication may recur in successive pregnancies, our case highlights the possibility of discordance for fetal goitre in siblings harbouring the same dyshormonogenesis-associated genetic mutations. Genetic ascertainment may facilitate prenatal diagnosis and assist management in familial cases. 


\section{Learning points:}

- $\mathrm{CH}$ due to biallelic, loss-of-function TG mutations is well-described and readily treatable in childhood however mechanical complications from associated fetal goitre may include polyhydramnios, neonatal respiratory compromise and neck hyperextension with dystocia complicating delivery.

- $\mathrm{CH}$ due to $T G$ mutations may manifest with variable phenotypes, even within the same kindred.

- Treatment options for hypothyroid dyshormogenic fetal goitre in a euthyroid mother include intraamniotic thyroid hormone replacement in cases with polyhydramnios or significant tracheal obstruction. Alternatively, cases may be managed conservatively with radiological surveillance, elective delivery and neonatal levothyroxine treatment, although intubation and ventilation may be required to support neonatal respiratory compromise.

- Genetic ascertainment in such kindreds may enable prenatal diagnosis and anticipatory planning for antenatal management of further affected offspring.

\section{Background}

Congenital hypothyroidism $(\mathrm{CH})$ is the commonest neonatal endocrine disorder, (incidence 1:1500 in Europe) and a national UK screening programme enables prompt diagnosis and initiation of levothyroxine treatment (1). Dyshormonogenesis, in which there is inadequate thyroid hormone biosynthesis due to a specific defect in one of the protein components of the thyroid hormone biosynthetic machinery, usually arises due to mutations in the following genes: TG, TPO, DUOX2, DUOXA2, IYD, SLC26A4 and SLC5A5. European Society for Paediatric Endocrinology (ESPE) guidelines recommend genetic counselling in such cases, although we have observed interregional variability in the proportion of cases undergoing molecular genetic ascertainment in the UK (2).

Significant fetal hypothyroid goitre is a rare consequence of fetal dyshormonogenesis, and complications include polyhydramnios due to oesophageal compression and malpresentation due to neck hyperextension at delivery; additionally, neonatal tracheal compression may cause fatal respiratory compromise $(3,4)$. The infrequent occurrence of non-autoimmune fetal hypothyroid goitre and the risks associated with intrauterine diagnosis and therapy have precluded large-scale studies of optimal treatment. Intraamniotic T4 may be beneficial in reducing goitre size however in the UK, parenteral T4 is not readily available, and there is one reported UK case in which intraamniotic T3 administered prior to T4 initiation achieved a successful outcome. The optimal dosing regimen for either drug remains to be ascertained $(2,4,5)$.

We describe the second case of hypothyroid fetal goitre to be treated with intraamniotic T3 injection whilst T4 was being sourced, and the resulting intrauterine death which highlights the fetal compromise which may occur in this context. Genetic ascertainment in the surviving sibling with dyshormonogenic $\mathrm{CH}$ showed that compound heterozygous $T G$ mutations were the likely aetiology for the fetal presentation, enabling appropriate genetic counselling to be initiated.

\section{Case presentation}

A male infant (Fig. 1: P1) weighing $3.58 \mathrm{~kg}$ was born at term to non-consanguineous, euthyroid Indian parents after an uncomplicated pregnancy. Following an instrumental delivery, he required resuscitation (Apgar scores were 5 (1 min) and 7 (5 min)) and sustained a depressed frontal bone and left sided facial nerve palsy due to birth trauma,

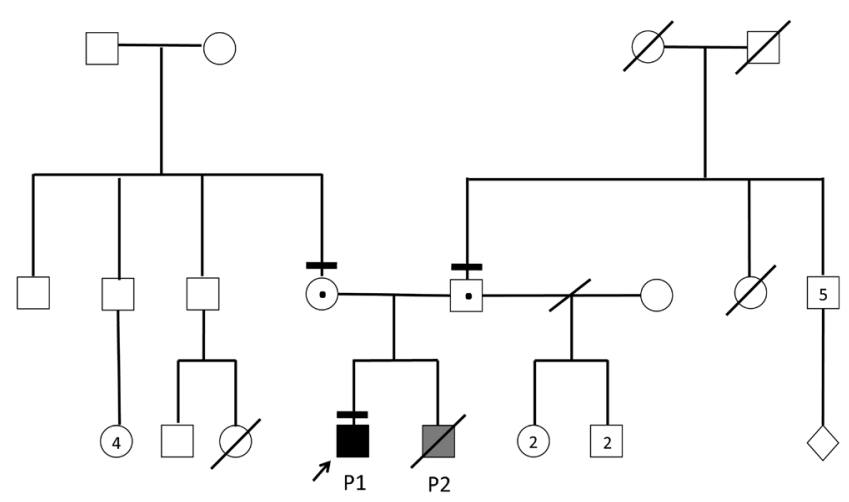

Figure 1

Pedigree diagram for the affected family. Black bars denote those individuals who have been genotyped; black and grey shading denotes confirmed (P1) and presumed (P2) compound heterozygous genoypes, heterozygotes for one of the two mutations are denoted by a central black dot. 
A

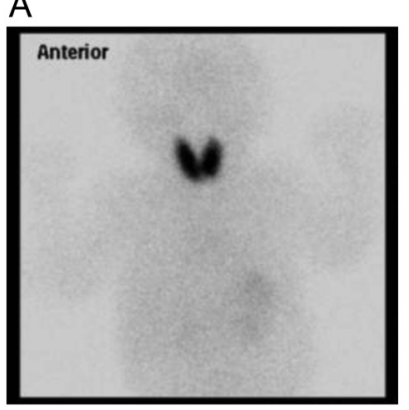

B

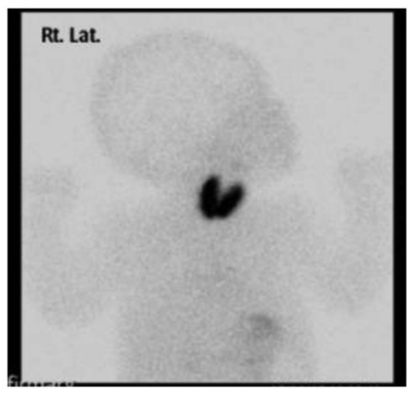

Figure 2

Technetium-99m pertechnetate thyroid scintigraphy performed in P1 aged 29 days, demonstrating appropriate radionuclide uptake in a normally-sited thyroid gland, consistent with dyshormonogenesis.

(A) Anterior and (B) lateral images.

which were conservatively managed. Neonatal screening revealed CH (fT4: 7 pmol/L normal range (NR): 9.0-25.0), Thyroid-stimulating hormone (TSH) $>100 \mathrm{mU} / \mathrm{L}$ (NR: 0.3-5.0)), and a subsequent isotope scan demonstrated appropriate uptake in a normally-sited thyroid gland (Fig. 2). He had prolonged jaundice but was otherwise healthy and prompt initiation of levothyroxine treatment has enabled normal growth and development.

There was no family history of thyroid disease however his mother exhibited congenital shortened right limbs; his father was well and had fathered 4 healthy children in a previous relationship (Fig. 1). They went on to have a second pregnancy complicated by a fetal goitre detected on routine ultrasound (Fig. 3).

\section{Investigation}

During the second pregnancy, a 20-week obstetric scan revealed a fetal thyroid goitre which continued to enlarge between $23+3$ and $29+2$ weeks, with associated polyhydramnios (Fig. 3). Maternal biochemistry confirmed euthyroidism (TSH: $3.2 \mathrm{mIU} / \mathrm{L}$; NR: 0.3-5.0) with absent autoantibodies (TPO <20 IU/mL; NR: 0-60), and amniotic fluid TSH measurements at $29+4$ weeks were consistent with fetal hypothyroidism: TSH: $1.5 \mathrm{mIU} / \mathrm{L}(\mathrm{NR}<0.51)$.

\section{Treatment}

The large hypothyroid fetal goitre and polyhydramnios supported a diagnosis of fetal dyshormonogenesis with obstructive dysphagia, due to local oesophageal compression. A decision was made to commence intraamniotic thyroid hormone replacement, initially by injection of $120 \mu \mathrm{g}$ T3 at $29+4$ weeks with concomitant amniocentesis $(260 \mathrm{~mL})$. T3 was selected in the first instance due to the fact that we encountered difficulties sourcing parenteral $\mathrm{T} 4$ in the UK and the requirement for local approval to be granted prior to its administration would have resulted in significant treatment delay had we opted for first-line T4 therapy. At 31 weeks gestation, considerable polyhydramnios and fetal hypothyroidism persisted (amniotic TSH: $5.3 \mathrm{mIU} / \mathrm{L}$ (NR $<0.51$ ), compared with TSH $1.5 \mathrm{mIU} / \mathrm{L}$ at $29+4$ weeks) therefore $150 \mu \mathrm{g}$ intraamniotic T4 was administered following further amniocentesis having been sourced and locally approved in the interim.

\section{Outcome and follow-up}

At $31+3$ weeks gestation, an obstetric scan was performed for reduced fetal movements, which revealed an intrauterine death in association with significant polyhydramnios including accumulation of amniotic fluid in the extraamniotic/subchorionic space. The stillborn infant (P2) was male, weighing $1.62 \mathrm{~kg}$, with a normal male karyotype on amniotic fluid analysis. There was no evidence of intrauterine infection and postmortem was declined.

Although DNA was not extracted from the fetus, the history of $\mathrm{CH}$ in the elder sibling prompted genetic screening of TPO (thyroid peroxidase) and $T G$ (thyroglobulin). Compound heterozygous $T G$ mutations were identified, comprising a novel, paternallyinherited single nucleotide deletion (c.5178delT, p.A1727Hfs*26), and a maternally-inherited missense mutation (c.7123G >A, p.G2375R), which has been

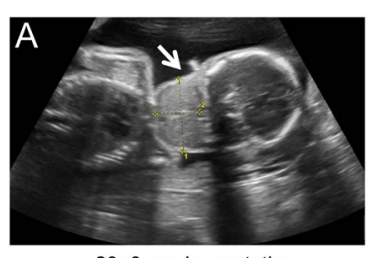

$23+3$ weeks gestation

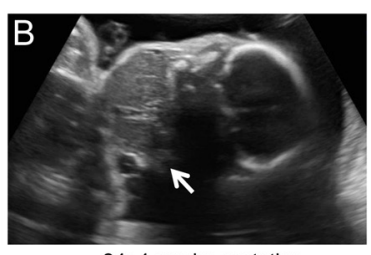

$24+4$ weeks gestation

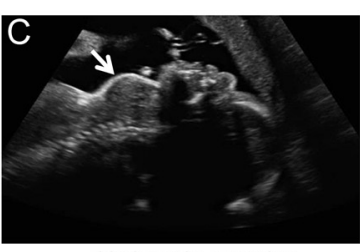

$29+2$ weeks gestation
Figure 3

Sequential fetal ultrasound images with gestational age annotated demonstrating progressive fetal goitre (white arrow). A coronal view at $23+3$ weeks gestation demonstrates a goitre measuring $42.8 \mathrm{~mm} \times 26.6 \mathrm{~mm}$ which persists aged $24+4$ weeks (sagittal view) and has enlarged to $54 \mathrm{~mm} \times 36 \mathrm{~mm} \times 52 \mathrm{~mm}$ aged $29+2$ weeks gestation (sagittal view), $48 \mathrm{~h}$ prior to intrauterine liothyronine therapy. 

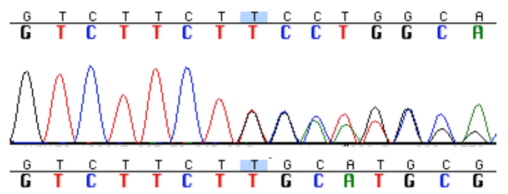

Proband
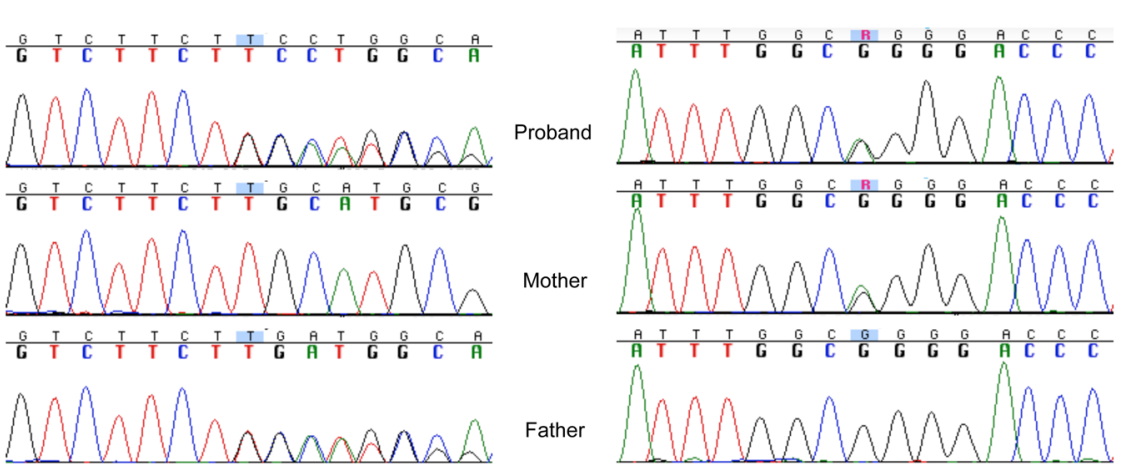

Mother

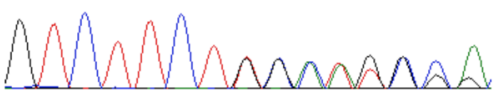

\section{Figure 4}

Sequencing electropherograms from the proband (P1), mother and father, illustrating compound heterozygous TG mutations, comprising a novel, paternally-inherited single nucleotide deletion (c.5178delT, p.A1727Hfs*26), and a maternallyinherited missense mutation (c.7123G $>$ A, p.G2375R). previously reported in association with $\mathrm{CH}$ without fetal goitre (6) Fig. 4).

\section{Discussion}

Although biallelic $T G$ mutations commonly underlie $\mathrm{CH}$ due to dyshormonogenesis, their absolute frequency is low, and fetal goitre is a rare complication which may be associated with significant morbidity $(3,7,8)$. The proband in this family harboured compound heteroygous $T G$ mutations, and although the stillborn fetus was not genotyped, his goitrous hypothyroidism in utero supports an identical genetic diagnosis. Although the proband did not have goitre, he exhibited biochemically moderatesevere $\mathrm{CH}$ at diagnosis, and such phenotype variability occurs in other kindreds harbouring TG mutations. The phenotypic modulators remain uncharacterized although dietary iodine intake, (not quantified here), may be implicated (9).

ESPE guidelines recommend genetic counselling in patients with dyshormonogenetic $\mathrm{CH}$, since most patients have an underlying genetic basis. Fetal goitre has been reported in association with $T G$ and TPO mutations however both discordance and recurrence in successive pregnancies has been documented $(2,3,10)$. A genetic diagnosis in the kindred enabled us to counsel the parents that future offspring had a $25 \%$ probability of inheriting biallelic mutations and developing $\mathrm{CH}$ with a consequent risk of fetal goitre. In subsequent pregnancies, prenatal genetic diagnosis could facilitate management by mandating stringent surveillance for fetal goitre, and optimization of maternal iodine status. Additionally, given the practical difficulties in obtaining parenteral T4 in the UK, this could be pre-emptively acquired in the event of a positive genetic diagnosis in the fetus, in case intraamniotic T4 therapy became necessary.

Management of hypothyroid fetal goitre remains controversial, as there are significant risks associated with both diagnostic amniocentesis/cordocentesis and intraamniotic thyroid hormone therapy including miscarriage. Although cordocentesis is the gold standard for assessment of fetal thyroid status, we felt the clinical likelihood of fetal hypothyroidism was high (euthyroid mother, negative autoantibodies and brother with $\mathrm{CH}$ ), and therefore measured amniotic fluid thyroid hormone levels since this could be achieved simultaneously with therapeutic amniocentesis and intraamniotic injection $(2,7)$.

Treatment of fetal hypothyroid goitre requires optimization of maternal thyroid status including minimizing doses of antithyroid drugs, followed by either conservative management with neonatal levothyroxine administration, or direct fetal treatment in utero with thyroid hormone, since the placenta is not readily permeable to maternally-administered thyroxine or liothyronine $(8,11)$. Fetal hypothyroid dyshormonogenic goitre in the context of maternal euthyroidism has been managed successfully with radiological surveillance, followed by elective delivery and neonatal levothyroxine treatment, especially in cases presenting at late gestational ages. However intubation and ventilation may be required to support neonatal respiratory compromise at birth, and concerns regarding dystocia may necessitate Caesarean section. MRI may be more effective than ultrasound in assessing the degree of tracheal compression $(8,12,13)$.

Cases treated in utero have usually been given intraamniotic levothyroxine, for which one retrospective cohort study supports a beneficial effect in reducing goitre size. Intraamniotic T3 (three injections of 60, 60 and $120 \mu \mathrm{g}$ at $30+5-32+3$ weeks) prior to $\mathrm{T} 4$ initiation has also been used successfully in another UK case. Treatment response is assessed by monitoring goitre size and resolution of polyhydramnios, sometimes in addition to sequential fetal thyroid hormone measurements. 3D ultrasonography may prove a superior tool to standard techniques in monitoring fetal goitre size in the future 
$(3,2,4,14)$. The optimal dosing regimen for intraamniotic therapy and effect on neonatal thyroid hormone status remains undefined, and associated complications include premature delivery, intrauterine infection and death. Published records describe preterm labour and chorioamnionitis following intraamniotic $\mathrm{T} 4$, and a case of fetal death is alluded to in one report (8, summarized in (15)). In view of these caveats, intraamniotic therapy is generally considered only when there is progressive hydramnios or likely tracheal occlusion, although conservative management in this context may also be associated with preterm delivery and neonatal death $(3,7,13,15)$.

In our patient, logistical barriers (sourcing parenteral T4 in the UK, obtaining local approval for therapy) initially precluded the use of intraamniotic T4. However worsening polyhydramnios compelled us to administer an intraamniotic injection of the active thyroid hormone T3 (which has a shorter half-life and greater potency than T4) until parenteral T4 became available. Treatment with intraamniotic $\mathrm{T} 4$ would have been preferable to $\mathrm{T} 3$, which will cause more precipitous fluctuations in hormone levels and has only one case report to support its use. The precise cause for the intrauterine death in our case was undertermined, although significant polyhydramnios with extension into the extraamniotic/subchorionic space was observed and is likely to have played a major role. Additionally, although there was no evidence of intrauterine infection, the treatment itself may have contributed to fetal demise, given the known risk of fetal loss associated with amniocentesis and the short time interval between intraamniotic injection and intrauterine death. At 31 weeks gestation, we selected a dose of intraamniotic T4 similar to that used in the previous intraamniotic T3/T4 combination therapy report $(150 \mu \mathrm{g})$ however others have reported successful outcomes with higher doses of T4 alone (200-400 $\mu \mathrm{g}$ at similar gestational age), raising the possibility this dose was insufficient $(4,5)$. Dysphagia due to tracheo-oesophageal compression may also have impaired the ability of the fetus to ingest intraamniotic medication such that that earlier initiation of thyroid hormone therapy may have been more effective (5).

Difficulties in obtaining parenteral $\mathrm{T} 4$ in the UK compound management of fetal goitrous hypothyroidism and our case emphasizes the requirement for further studies before T3 is considered a suitable therapeutic alternative. Additionally, our report highlights the morbidity and mortality associated with hypothyroid fetal goitre and its intrauterine treatment, which may be obscured in the literature due to a tendency for under-reporting adverse outcomes in similar complex cases (8). Although not universally undertaken, genetic ascertainment in such families may help predict risk of recurrence in future pregnancies and guide antenatal management.

\section{Declaration of interest}

The authors declare that there is no conflict of interest that could be perceived as prejudicing the impartiality of the research reported.

\section{Funding}

This work was supported by funding from the Wellcome Trust (grant number $100585 / Z / 12 / Z$ to N S) and the National Institute for Health Research Biomedical Research Centre Cambridge (N S). The Genomics/ Transcriptomics Core Facility is supported by the UK Medical Research Council (MRC) Metabolic Disease Unit (MRC_MC_UU_12012/5) and a Wellcome Trust Strategic Award (100574/Z/12/Z).

\section{Patient consent}

We confirm that written informed consent has been obtained from the patients' mother for publication of the submitted article and accompanying images.

\section{Author contribution statement}

N S initiated genetic studies in the patient and wrote the manuscript, A K N performed the genetic studies, I S oversaw obstetric management, J G oversaw endocrine management and C P, P V and S-M P oversaw clinical genetic management of the patients. The authors acknowledge the Genomics and Transcriptomics Core Facility at the Wellcome Trust-MRC Institute of Metabolic Science for assistance with Sanger sequencing.

\section{References}

1 Persani L 2012 Congenital hypothyroidism with gland in situ is more frequent than previously thought. Frontiers in Endocrinology $\mathbf{3}$ Article 18. (doi:10.3389/fendo.2012.00018)

2 Leger J, Olivieri A, Donaldson M, Torresani T, Kriude H, van Vliet G, Polak M, Butler G, on behalf of ESPE-PES-SLEP-JSPE-APEG-APPESISPAE \& The Congenital Hypothyroidism Consensus Conference Group 2014 European Society for Paediatric Endocrinology consensus guidelines on screening, diagnosis and management of congenital hypothyroidism. Journal of Clinical Endocrinology and Metabolism 99 363-384. (doi:10.1210/jc.2013-1891)

3 Ghazi A-A M, Ordookhani A, Pourafkari M, Fallahian M, Bahar A, Hedayati M, Hafizi A \& Azizi F 2005 Intrauterine diagnosis and management of fetal goitrous hypothyroidism: a report of an Iranian family with three consecutive pregnancies complicated by fetal goiter. Thyroid 15 1341-1347. (doi:10.1089/thy.2005.15.1341)

4 Ribault V, Castanet M, Bertrand, A-M, Guibourdenche J, Vuillard E, Luton D, Polak M \& The French Fetal Goiter Study Group 2009 Experience with intraamniotic thyroxine treatment in nonimmune fetal goitrous hypothyroidism in 12 cases. Journal of Clinical Endocrinology and Metabolism 94 3731-3739. (doi:10.1210/jc.20082681)

5 Agrawal P, Ogilvy-Stuart A \& Lees C 2002 Intrauterine diagnosis and management of congenital goitrous hypothyroidism. Ultrasound 
in Obstetrics and Gynecology 19 501-505. (doi:10.1046/j.14690705.2002.00717.x)

6 Hishinuma A, Fukata S, Nishiyama S, Nishi Y, Oh-Ishi M, Murata Y, Ohyama Y, Matsuura N, Kasai K, Harada S, et al. 2006 Haplotype analysis reveals founder effects of thyroglobulin gene mutations C1058R and C1977S in Japan. Journal of Clinical Endocrinology and Metabolism 91 3100-3104. (doi:10.1210/jc.2005-2702)

7 Polak M \& Luton D 2014 Fetal thyroidology. Best Practice and Research Clinical Endocrinology and Metabolism 28 161-173. (doi:10.1016/j. beem.2013.04.013)

8 Reynolds BC, Simpson JH, Macara L, Watt AJB, Kubba H, Donaldson MDC \& Pohlenz J 2006 Goitrous congenital hypothyroidism in a twin pregnancy causing respiratory obstruction at birth: implications for management. Acta Pediatrica 95 1345-1348. (doi:10.1080/08035250600711074)

9 Pardo V, Rubio IGS, Knobel M, Aguiar-Oliveira MH, Santos MM, Gomes SA, Oliveira CRP, Targovnik HM \& Medeiros-Neto G 2008 Phenotypic variation among four family members with congenital hypothyroidism caused by two distinct thyroglobulin gene mutations. Thyroid 18 783-787. (doi:10.1089/thy.2007.0321)

10 Borgel K, Pohlenz J, Holzgreve W \& Bramswig JH 2005 Intrauterine therapy of goitrous hypothyroidism in a boy with new compound heterozygous mutation (Y453D ad C800R) in the thyroid peroxidase gene. A long-term follow-up. American Journal of Obstetrics and Gynecology 193 857-858. (doi:10.1016/j. ajog.2005.01.060)

11 Davidson KM, Richards DS, Schatz DA \& Fisher DA 1991 Successful in utero treatment of fetal goiter and hypothyroidism. New England Journal of Medicine 324 543-546. (doi:10.1056/ NEJM199102213240807)

12 Bae JY, Joo LH, Jung JE \& Hong SY 2015 Prenatal diagnosis of fetal goiter in a euthyroid mother. Korean Journal of Perinatology 26 365-368. (doi:10.14734/kjp.2015.26.4.365)

13 Gungoren A, Dolapcioglu K, Hakverdi AU, Balci A \& Guzelmansur I 2010 Fetal goiter in the absence of maternal thyroid disease: a case report. Perinatal Journal 18 101-104.

14 Kim MJ, Chae Y-H, Park SY \& Kim MY 2016 Intra-amniotic thyroxine to treat fetal goiter. Obstetrics and Gynecology Science 59 66-70. (doi:10.5468/ogs.2016.59.1.66)

15 Stoppa-Vaucher S, Francoeur D, Grignon A, Alos N, Pohlenz J, Hermanns P, Van Vliet G \& Deladoëy J 2010 Non-immune goiter and hypothyroidism in a 19-week old fetus: a plea for conservative treatment. Journal of Pediatrics 156 1026-1029. (doi:10.1016/j. jpeds.2010.01.018)

Received in final form 26 April 2017

Accepted 10 May 2017 